\title{
Single fraction high-dose-rate brachytherapy as monotherapy for low and intermediate risk prostate cancer: toxicities and early outcomes from a single institutional experience
}

\author{
Justin M. Barnes, MS', Prashant Gabani, MD², Max Sanders ${ }^{3}$, Anupama Chundury, MD4, Michael Altman, PhD, DABR², \\ Jose Garcia-Ramirez, MS², Harold Li, PhD², Jacqueline E. Zoberi, PhD², Brian C. Baumann, MD², Hiram A. Gay, MD² \\ ISaint Louis University School of Medicine, Saint Lovis, United States, ${ }^{2}$ Department of Radiation Oncology, Washington University School of \\ Medicine, Saint Louis, United States, ${ }^{3}$ University of Missouri-St. Lovis, Saint Lovis, United States, ${ }^{4}$ Department of Radiation Oncology, Rutgers \\ Robert Wood Johnson Medical School, New Brunswick, United States
}

\begin{abstract}
Purpose: High-dose-rate brachytherapy (HDR-BT) delivered in a single fraction as monotherapy is a potential treatment modality for low- and intermediate-risk prostate cancer (LIR-PC); however, outcome data with this technique remain limited. Here we describe our institutional HDR monotherapy experience and report the efficacy and toxicity of this treatment.

Material and methods: LIR-PC patients who received a definitive single fraction HDR-BT during 2013-2017 were retrospectively identified. The intended HDR monotherapy dose was $19 \mathrm{~Gy}$ in one fraction. Acute (<90 days) and late ( $\geq 90$ days) toxicity was assessed using CTCAE version 4.03. Trends in prostate-specific antigen (PSA) and American Urological Association (AUA) symptom scores after treatment were assessed using Bayesian linear mixed models. The Kaplan-Meier method was used to evaluate biochemical failure-free survival (BFFS).

Results: 28 patients with median follow-up of 23.6 months were identified. The median age at treatment was 65 years (48-83). The NCCN risk groups were low in 14, favorable intermediate in 10, and unfavorable intermediate in 4 patients. There were $5(18 \%)$ and $0(0 \%)$ acute grade 2 genitourinary (GU) and gastrointestinal (GI) toxicities, respectively, and one (4\%) acute grade $3 \mathrm{GU}$ toxicity. There were no late grade 3 toxicities, and $5(18 \%)$ and $0(0 \%)$ late grade 2 GU and GI toxicities respectively. PSA values and AUA symptom scores decreased significantly after treatment. There were 3 biochemical failures with the two- and three-year BFFS of $90.7 \%$ and $80.6 \%$, respectively.

Conclusions: Early results from a single institution suggest that single fraction HDR-BT with 19 Gy has limited toxicity, although with suboptimal biochemical control.

Key words: prostate cancer, brachytherapy, high-dose-rate, single fraction.

\section{Purpose}

Treatment options for prostate cancer include active surveillance, radical prostatectomy, and radiation therapy (RT), including external beam RT, low-dose-rate (LDR) brachytherapy (BT) and high-dose-rate (HDR) BT. Historically, conventionally fractionated external beam RT, typically delivered over 20 to 44 fractions, has proven to be an effective treatment for low and intermediate risk prostate cancer. Recent RT dose escalation studies have showed overall survival of approximately $70 \%$ and biochemical failure ranging from approximately $17 \%$ to $40 \%$ at 10 years $[1,2,3,4,5]$. HDR-BT and LDR-BT are forms of internal RT that avoid variations in internal anatomy and inaccuracy in daily setup while offering shortened treatment duration and favorable dosimetry with no need to expand the clinical target volume (CTV), and thus have advantages to external beam RT $[6,7,8]$.

In the late 1980s, it was determined that the actual RT dose coverage of LDR-BT was lower than what was expected in the pre-planning stage, so investigators began exploring the use of HDR-BT, the rationale being that post-implant RT planning and the higher energy isotope, ${ }^{192} \mathrm{Ir}$, would enable highly conformal dose delivery to the periphery of the prostate to maximize tumor coverage 
while minimizing the dose to the rectum and bladder [7,9]. The first reported series of patients treated with HDR-BT by Galalae et al. as a boost to external beam RT showed excellent results, as did long-term follow-up [10,11,12]. In 1995, HDR-BT delivered in multiple fractions as monotherapy for prostate cancer became an investigational option [13]. Since that time, HDR-BT monotherapy has been shown to be a well-tolerated and efficacious treatment for low to intermediate risk prostate cancer [6]. While hypofractionated schedules have been investigated, no consensus on the best regimen has been established [7] However, due to the disadvantages of HDR-BT such as potentially two or more implants as well as multiple fractions that must be separated by more than 6 hours, which may increase procedure-related adverse events and inconvenience for the patient [7], an ultra-hypofractionated - specifically one fraction - schedule may be desirable. Such a regimen may make HDR-BT favorable to LDR-BT given its practical, physical, and biological advantages including no use of a free live source, no risk for source loss, no need for radioprotection precautions after discharge, relative ease of treating extra-prostatic extension and seminal vesicle involvement, dosimetry that can be adjusted and known prior to radiation exposure, and a favorable $\alpha / \beta$ ratio for hypofractionation [8].

HDR-BT in a single fraction as monotherapy for prostate cancer was first described in 2012 [14], and early data suggest that it results in similar biochemical control and at least as favorable a toxicity profile as other treatments $[14,15,16,17,18,19,20,21,22]$. Given limited data, our objective was to evaluate the toxicities and early outcomes of patients treated with single fraction HDR-BT monotherapy for low and intermediate risk prostate cancer at a single institution.

\section{Material and methods}

\section{Participants and HDR-BT procedure}

Consecutive patients with low and intermediate risk prostate cancer (LIR-PC) who received definitive HDR monotherapy in a single fraction during 2013-2017 at a single institution were retrospectively identified after approval from the Institutional Review Board. All patients received no other definitive therapy, and only one received concurrent androgen deprivation therapy (ADT) due to unfavorable-intermediate risk. The other patients with unfavorable-intermediate risk declined ADT. Patient selection for HDR monotherapy included the following criteria: histologically confirmed LIR-PC defined by NCCN classification, no evidence of nodal or metastatic disease, and no prior RT to the prostate or pelvis. Patients had the following imaging: magnetic resonance imaging (MRI; multiparametric) only: 12; computed tomography (CT) only: 2; bone scan only: 1; MRI and bone scan: 2; CT and bone scan: 1; MRI and NaF positron emission tomography (PET): 1 ; and MRI, CT, and bone scan: 1; no imaging: 1 . One patient reportedly had an MRI or CT but we could not confirm it. After induction of general anesthesia, HDR catheters were inserted under transrectal ultrasound guidance, with an average of
12 catheters at the periphery of the prostate and 4 central catheters surrounding the urethra, with attention to cover the base of the prostate and proximal seminal vesicles. The proximal seminal vesicles were usually covered with the 4 most posterior catheters. Intraoperative cystoscopy was used to confirm tenting of the bladder as a surrogate to confirm catheter placement at the base of the prostate. Additionally, cystoscopy also confirmed the absence of treatment catheters within the urethra or bladder, and catheters were adjusted as necessary. Implantation of the seminal vesicles was visualized using the transrectal ultrasound and confirmed with CT. At the end of the procedure, a Foley catheter was placed. The patients then underwent a CT scan to verify catheter placement, and adjustment of the catheters was made as needed. The intended HDR monotherapy dose was 19 Gy in one fraction, though the dose was increased in mid-2017 to $21 \mathrm{~Gy}$ following reports of suboptimal control with 19 Gy [17] and a report describing use of higher doses [16]. HDR treatment was delivered about 3-4 hours after the catheter placement. For the 19 Gy regimen, the dose constraints used were: prostate $V_{100}>90 \%$, urethra $\mathrm{V}_{125}<1 \mathrm{cc}$, urethra $\mathrm{V}_{150}=0 \mathrm{cc}$, bladder and rectum $\mathrm{V}_{75}$ $<1 \mathrm{cc}$, and bladder and rectum $\mathrm{V}_{150}=0 \mathrm{cc}$. For the $21 \mathrm{~Gy}$ regimen, the dose constraints were chosen to be similar to the 19 Gy constraints and were: prostate $V_{100}>90 \%$, urethra $\mathrm{V}_{115}<1 \mathrm{cc}$, urethra $\mathrm{V}_{135}=0 \mathrm{cc}$, bladder and rectum $\mathrm{V}_{70}<1 \mathrm{cc}$, and bladder and rectum $\mathrm{V}_{135}=0 \mathrm{cc}$. Our practice has not been to expand the gross tumor volume (GTV) to create a CTV.

The implant was removed after the delivery of radiotherapy (RT) and the patients were discharged home the same day. The Foley catheter was removed and a voiding trial was conducted. If the patient was unable to void, the Foley catheter was re-inserted; it was to be removed prior to, or at their 1-week follow-up as indicated. After treatment, patients were followed at regular intervals, and symptoms/toxicities and prostate-specific antigen (PSA) values were monitored. Acute $(<90$ days) and late $(\geq 90$ days) toxicity was assessed using the Common Terminology Criteria for Adverse Events (CTCAE) v4.03. A baseline American Urological (AUA) Symptom Score was assessed prior to treatment and obtained at follow-up visits.

\section{Data analysis}

Characteristics of the study population and acute and late toxicities are described using summary statistics. PSA and AUA post-treatment trends were assessed using Bayesian generalized linear mixed models, which facilitate probabilistic inference while accounting for the correlation between the multiple values obtained for each patient, with flexible quadratic and cubic time components to accurately model trends over time. The Kaplan-Meier method was used to estimate biochemical failure-free survival (BFFS), local failure-free survival (LFFS), regional failure-free survival (RFFS), distant failure-free survival (DFFS), and overall survival (OS). As this is a retrospective study without a date of randomization, the start date for time-to-event data was considered the date of HDR-BT. The log-rank test was used to compare surviv- 
al curves. Differences in BFFS were compared by NCCN version 2019 risk groups. All $P$-values are 2 -sided if applicable, and $p<0.05$ is considered statistically significant. Probabilities derived from Bayesian models are considered statistically significant when $>0.975$ (corresponding to a 2 -sided $p$-value $<0.05$ of the null hypothesis).

\section{Results}

A total of 28 patients with LIR-PC were retrospectively identified. Characteristics of the study population are given in Table 1. Median follow-up time was 23.6 months (0.3-59). The median age of treatment was 65 years (48-83). The NCCN risk groups were "very low" in 4, "low" in 10, "favorable-intermediate" in 10 patients, and "unfavorable-intermediate" in 4 patients. The median PSA at diagnosis was 6.0 (1.1-13.3), median AUA score prior to treatment was 12 (2-29), and median prostate volume at implant was 34.3 cc (16.5-122).

Table 1. Characteristics of study population

\begin{tabular}{|c|c|}
\hline \multicolumn{2}{|l|}{ Follow-up (months) } \\
\hline Median (range) & $23.6(0.3-59)$ \\
\hline \multicolumn{2}{|l|}{ Age (years) } \\
\hline Median (range) & $65(48-83)$ \\
\hline \multicolumn{2}{|l|}{ Race } \\
\hline Caucasian & 19 \\
\hline African American & 9 \\
\hline \multicolumn{2}{|l|}{ BMI } \\
\hline Median (range) & $27.8(22-49)$ \\
\hline \multicolumn{2}{|l|}{ KPS } \\
\hline 70 & 2 \\
\hline 90 & 17 \\
\hline 100 & 9 \\
\hline \multicolumn{2}{|l|}{ Presenting AUA symptom score } \\
\hline Median (range) & $12(2-29)$ \\
\hline \multicolumn{2}{|l|}{ Presenting PSA } \\
\hline Median (range) & $5.93(1.1-13.3)$ \\
\hline \multicolumn{2}{|l|}{ T stage } \\
\hline T1c & 25 \\
\hline $\mathrm{T} 2 \mathrm{a}$ & 3 \\
\hline \multicolumn{2}{|l|}{ Gleason score } \\
\hline $3+3$ & 16 \\
\hline $3+4$ & 8 \\
\hline $4+3$ & 4 \\
\hline \multicolumn{2}{|l|}{ \# Cores positive } \\
\hline Median (range) & $3(1-9)$ \\
\hline \multicolumn{2}{|l|}{ NCCN risk group } \\
\hline Very low & 4 \\
\hline Low & 10 \\
\hline Favorable intermediate & 10 \\
\hline Unfavorable intermediate & 4 \\
\hline
\end{tabular}

BMI - body mass index, KPS - Karnofsky Performance Status, AUA - American Urological Association, LDR - low-dose-rate, HDR - high-dose-rate, ADT - androgen deprivation therapy.
One patient experienced an acute grade 3 toxicity, experiencing urinary retention at 2 months after BT (Table 2). Four patients (14\%) experienced an acute grade 2 urinary toxicity, with $5(18 \%)$ reporting urinary frequency, $3(11 \%)$ reporting urinary urgency, $1(4 \%)$ reporting dysuria, and $2(7 \%)$ reporting urinary retention. Five patients $(18 \%)$ required a Foley catheter to be placed in the immediate postoperative period; it was removed by before or at their one-week post-treatment follow-up. There were no late grade $\geq 3$ toxicities (Table 2). Five patients $(18 \%)$ reported late grade 2 toxicities, with all $5(18 \%)$ reporting urinary frequency, $3(11 \%)$ reporting urinary urgency, 1 (4\%) reporting dysuria, and 1 (4\%) reporting urinary retention. No patients experienced gastrointestinal (GI) toxicities.

American Urological Association symptom scores prior to and after HDR-BT are given in Figure 1. Prior to treatment, $13(57 \%)$ patients had moderate (AUA symptom score $8-19)$ and 4 (17\%) had severe (AUA symptom

\begin{tabular}{|c|c|}
\hline \multicolumn{2}{|c|}{ American Brachytherapy Society LDR Criteria } \\
\hline AUA score > 15 & 7 \\
\hline Prostate volume $<20 \mathrm{cc}$ & 3 \\
\hline Prostate volume $>60 \mathrm{cc}$ & 3 \\
\hline Any of above criteria & 12 \\
\hline \multicolumn{2}{|l|}{ Prostate volume (treatment) } \\
\hline Median (range) & $35.2(16.5-122)$ \\
\hline \multicolumn{2}{|l|}{ Prostate $\mathrm{V}_{90}$} \\
\hline Median (range) & $98.5(94.5-99.9)$ \\
\hline \multicolumn{2}{|l|}{ Prostate $\mathrm{V}_{95}$} \\
\hline Median (range) & $97.2(92.0-99.5)$ \\
\hline \multicolumn{2}{|l|}{ Prostate $\mathrm{V}_{100}$} \\
\hline Median (range) & $95.3(89-98.6)$ \\
\hline \multicolumn{2}{|l|}{ Bladder $\mathrm{V}_{75}$} \\
\hline Median (range) & $0(0-0.52)$ \\
\hline \multicolumn{2}{|l|}{ Rectum $V_{75}$} \\
\hline Median (range) & $0.24(0-0.8)$ \\
\hline \multicolumn{2}{|l|}{ Urethra $\vee_{125}$} \\
\hline Median (range) & $0.03(0-0.45)$ \\
\hline \multicolumn{2}{|l|}{ Dose of HDR } \\
\hline 19 Gy & 26 \\
\hline $21 \mathrm{~Gy}$ & 2 \\
\hline \multicolumn{2}{|l|}{ Bladder punctured } \\
\hline No & 17 \\
\hline Yes & 11 \\
\hline \multicolumn{2}{|l|}{ ADT } \\
\hline Yes & 1 (short-term) \\
\hline No & 26 \\
\hline \multicolumn{2}{|l|}{ Time to PSA nadir (months) } \\
\hline Median (range) & $12.1(1.6-34)$ \\
\hline
\end{tabular}


Table 2. Acute and late toxicities

\begin{tabular}{|c|c|c|c|c|}
\hline \multirow[t]{2}{*}{ Toxicity } & \multicolumn{4}{|c|}{ Grade } \\
\hline & 0 & 1 & 2 & 3 \\
\hline \multicolumn{5}{|l|}{ Acute toxicities } \\
\hline Any acute symptom (max.) & $6(21 \%)$ & $17(61 \%)$ & $4(14 \%)$ & $1(4 \%)$ \\
\hline Frequency & $14(50 \%)$ & $9(32 \%)$ & $5(18 \%)$ & 0 \\
\hline Urgency & $21(75 \%)$ & $4(14 \%)$ & $3(11 \%)$ & 0 \\
\hline Dysuria & $25(89 \%)$ & $2(7 \%)$ & $1(4 \%)$ & 0 \\
\hline Retention & $22(79 \%)$ & $3(11 \%)$ & $2(7 \%)$ & $1(4 \%)$ \\
\hline Incontinence & $25(89 \%)$ & $3(11 \%)$ & 0 & 0 \\
\hline Hematuria & $12(43 \%)$ & $16(57 \%)$ & 0 & 0 \\
\hline Diarrhea & 0 & 0 & 0 & 0 \\
\hline Rectal pain & 0 & 0 & 0 & 0 \\
\hline Proctitis & 0 & 0 & 0 & 0 \\
\hline \multicolumn{5}{|l|}{ Late toxicities } \\
\hline Any late symptom (max.) & $10(36 \%)$ & $13(46 \%)$ & $5(18 \%)$ & 0 \\
\hline Frequency & $14(50 \%)$ & $9(32 \%)$ & $5(18 \%)$ & 0 \\
\hline Urgency & $18(64 \%)$ & $7(25 \%)$ & $3(11 \%)$ & 0 \\
\hline Dysuria & 27 (96\%) & 0 & $1(4 \%)$ & 0 \\
\hline Retention & $18(64 \%)$ & $9(32 \%)$ & $1(4 \%)$ & 0 \\
\hline Incontinence & $23(82 \%)$ & $5(18 \%)$ & 0 & 0 \\
\hline Hematuria & 27 (96\%) & $1(4 \%)$ & 0 & 0 \\
\hline Diarrhea & $28(100 \%)$ & 0 & 0 & 0 \\
\hline Rectal pain & 28 (100\%) & 0 & 0 & 0 \\
\hline Proctitis & 28 (100\%) & 0 & 0 & 0 \\
\hline
\end{tabular}

score $\geq 20)$ symptoms. After treatment, $12(60 \%)$ and $2(10 \%)$ at 12 months and $5(46 \%)$ and $2(14 \%)$ at 24 months had moderate or severe symptoms, respectively. Only 5 patients had follow-up with AUA scores at 36 months, and $80 \%$ and $0 \%$ had moderate and severe symptoms, respectively. Compared to pre-treatment AUA scores, $14(61 \%$ of those with available data) had increases in their AUA score at their 1-week follow-up visit. At 12 months and 24 months after the HDR-treatment, 6 and 5 (27\% and 31\%, respectively, of those with available data) had higher AUA scores than baseline (or increasing values if baseline AUA score was missing). Per the Bayesian model, there was no evidence that the AUA score increased following treatment, as the probability of increase was 0.817. AUA scores decreased significantly over time on average, as the probabilities that the AUA score had decreased from the post-treatment values at 12 and 24 months after treatment were 0.996 and 0.979 , respectively, according to the Bayesian model.

PSA trends following HDR-BT are given in Figure 2. The PSA value decreased significantly over time, where the probabilities that the 12- and 24-month PSA values were less than at treatment were 1.000 and 0.99997, respectively, according to the Bayesian model. At the last follow-up, three patients experienced biochemical failure, all in the 19 Gy group, with an associated two- and three-year biochemical failure-free survival (BFFS) of 90.7\% (95\% CI: 79.2-100) and 80.6\% (95\% CI: 61.7-100), respectively (Figure 3). There was one local failure, one biochemical failure with negative prostatic MRI and no biopsy obtained, one regional failure with negative prostate biopsy but choline-11 PET-avid pelvic lymph node (SUV $\left.\max _{\text {max }} 4.2\right)$, and one local plus distant failure with choline PET showing uptake in the left lateral base of the prostate as well as osseous metastatic disease (Table 3). Three-year local failure-free survival (LFFS), regional failure-free survival (RFFS), and distant failure-free survival (DFFS) were 85.7\% (95\% CI: 68.3-100), 90.0\% (95\% CI: 73.2-100), and 95.2\% (95\% CI: 86.6-100), respectively. There were no deaths. There was no difference in BFFS by risk group $(p=0.52)$, with three-year estimates of $80.0 \%$ (95\% CI: 


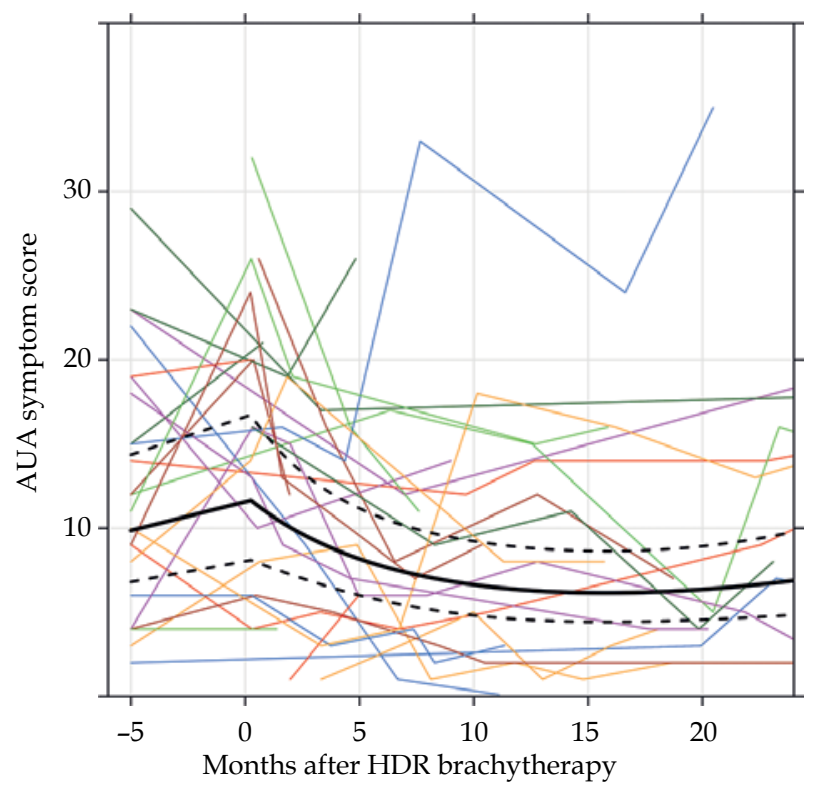

Fig. 1. Trends in AUA symptom score. The thin, colored lines represent individual patients' trajectories. The black lines represent the Bayesian model fit, with the dark line being the estimated mean and the dotted lines being the $95 \%$ credible interval. Note that all pre-treatment AUA scores are plotted at -5 months for illustrative purposes

A

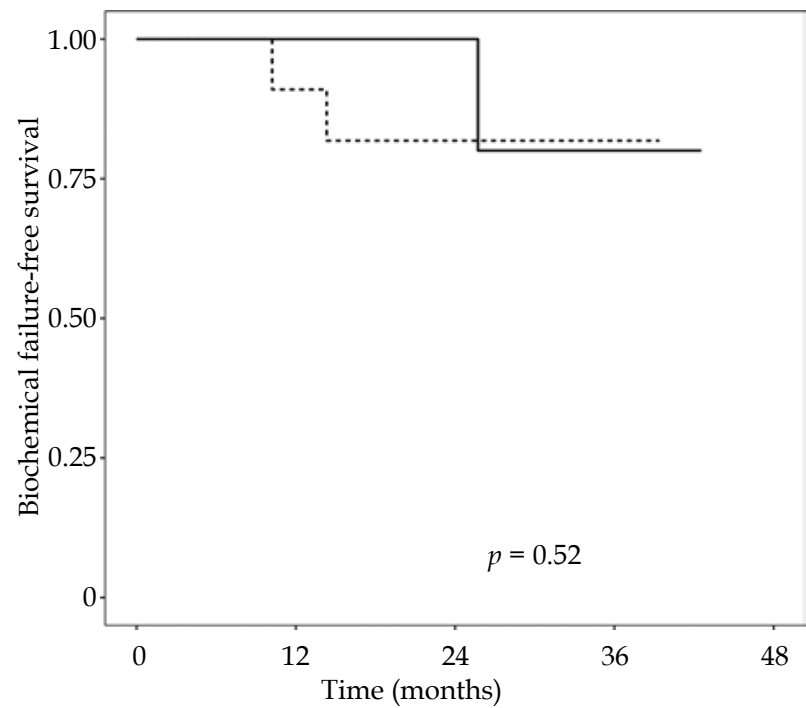

Number at risk

$\begin{array}{llllll}\text { IR } & 14 & 10 & 5 & 3 & 0 \\ \text { LR } & 14 & 10 & 7 & 2 & 1\end{array}$

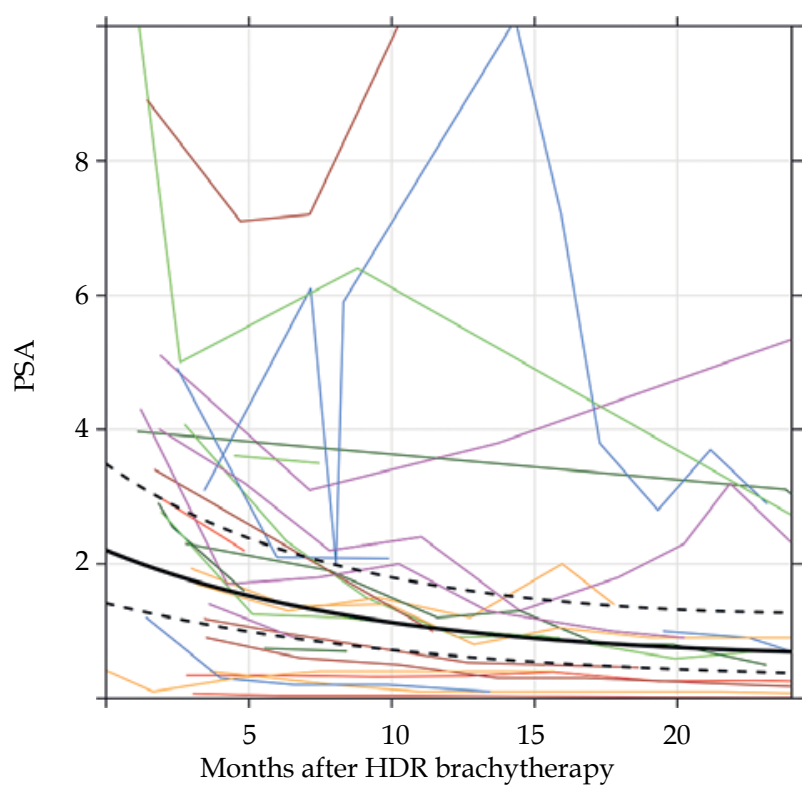

Fig. 2. Trends in prostate-specific antigen (PSA). The thin, colored lines represent individual patients' trajectories. The black lines represent the Bayesian model fit, with the dark line being the estimated mean and the dotted lines being the $95 \%$ credible interval

\section{B}

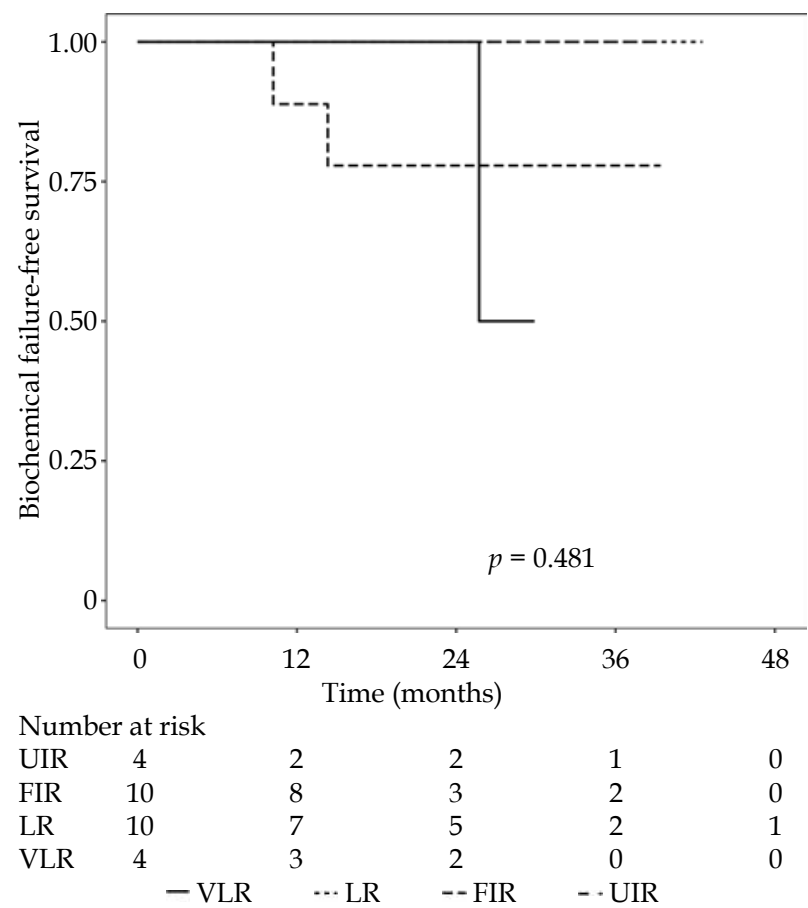

Fig. 3. Kaplan-Meier curves of biochemical failure-free survival by risk group. A) LR - low risk, IR - intermediate risk. B) VLR - very low risk, LR - low risk, FIR - favorable intermediate risk, UIR - unfavorable intermediate risk

51.6-100) and $81.8 \%$ (95\% CI: 61.9-100) in low and intermediate risk groups (Figure 3A). There was also no difference in BFFS when risk groups were stratified into very low, low, favorable-intermediate, and unfavorable-intermediate $(p=0.48$; Figure 3B).

\section{Discussion}

This study is one of a limited number of published series of patients receiving single-fraction HDR-BT as monotherapy for LIR-PC. Our results suggest that single 
Table 3. Characteristics of patients with failures

\begin{tabular}{|c|c|c|c|}
\hline & Patient 1 & Patient 2 & Patient 3 \\
\hline Type of failure & Biochemical failure & $\begin{array}{l}\text { Local + regional failure } \\
\text { (see Figure } 4 \mathrm{~A})\end{array}$ & $\begin{array}{l}\text { Local + distant failure } \\
\text { (see Figures } 4 \mathrm{~B} \text { and } 4 \mathrm{C} \text { ) }\end{array}$ \\
\hline Location of failure(s) & NA (no PET performed) & $\begin{array}{l}\text { Left anterior prostate } \\
\text { and peri-rectal LN }\end{array}$ & $\begin{array}{l}\text { Left lateral prostate, osseous metastases } \\
\text { of acetabulum, sternum, and C2 }\end{array}$ \\
\hline NCCN risk group & Favorable intermediate & Very low & Favorable intermediate \\
\hline Age at HDR-BT & 59 & 48 & 66 \\
\hline Presenting PSA & 6 & 4.3 & 13 \\
\hline Gleason score & $3+4$ & $3+3$ & $3+3$ \\
\hline Prostate volume (cc) & 36.6 & 20.2 & 16.48 \\
\hline Prostate $D_{90}$ & 21.4 & 18.7 & 20.1 \\
\hline Prostate $\mathrm{V}_{100}$ & 97.44 & 89 & 93.63 \\
\hline Bladder $V_{75}$ & 0 & 0 & 0.05 \\
\hline Rectum $V_{75}$ & 0.02 & 0 & 0.8 \\
\hline Urethra $\vee_{125}$ & 0.01 & 0.2 & 0.18 \\
\hline C-11 PET LF $V_{100}$ & NA & 60.3 & 49.5 \\
\hline C-11 PET LF D 90 & NA & 13.9 & 9.3 \\
\hline Months to biochemical failure & 14.3 & 25.7 & 10.2 \\
\hline Months to other failure & NA & 35.2 & 11.5 \\
\hline
\end{tabular}

HDR-BT - high-dose-rate brachytherapy, LN-lymph node, LF-local failure

fraction HDR-BT as monotherapy for prostate cancer has a favorable side-effect profile, though early biochemical failure estimates from our small cohort are somewhat higher than anticipated. To our knowledge, this is one of the few studies to quantitatively examine through a statistical model trends in PSA and symptom scores. Our results suggest that the AUA symptom score does not increase significantly after treatment, and it decreases over time compared to the immediate post-treatment scores, and PSA decreases significantly following treatment. However, a significant proportion of patients did not achieve PSA levels < 0.5 within 18 months, which has been found to be associated with higher risks of biochemical failure in patients receiving HDR-BT boost following external beam radiation therapy (EBRT) [23].

Other studies examining single fraction HDR brachytherapy as monotherapy for LIR-PC have demonstrated similar GU toxicities to our study (Table 4), with estimates of grade 2 or higher acute toxicity rates ranging from $0 \%$ to $51 \%$ and late toxicity rates ranging from $0 \%$ to $30 \%[14,17,18,19,20,21,22]$. There is one other report of grade $3 \mathrm{GU}$ toxicities, which, similarly to the current study, demonstrated a low prevalence of approximately $1.5 \%$ [19]. In regards to GI toxicities, in our study, we found no acute or late GI toxicity. This finding is consistent with the results of other studies evaluating HDR monotherapy, which have found grade 2 or higher GI toxicities ranging from $0 \%$ to $6 \%[14,17,18,19,20,21,22]$. The only report of a grade 3 GI toxicity was a late effect for one patient $(<1.5 \%)$ [22]. Two studies have evaluated single fraction HDR monotherapy in patients with locally advanced prostate cancer, with higher rates of acute grade 3 GU toxicities [15] but favorable long-term toxicities [16]. Studies of LDR-BT have shown somewhat higher rates of grade 3 toxicities than seen in HDR-BT [24].

Early results from our small cohort of patients suggested two- and three-year rates of BFFS of approximately $91 \%$ and $81 \%$ respectively, which are fairly consistent with prior studies of single fraction HDR-BT but less than ideal relative to the five-year BFFS of $85-94 \%$ observed with LDR as monotherapy (Table 4$)[17,18,20,24]$. For example, in the single fraction HDR study by Krauss et al., the authors found a slightly higher 3-year BFFS of 93.2\% [20]. However, with longer follow-up, Prada et al. and Siddiqui et al. reported a poor BFFS with 19 Gy $(66 \%$ at 6 years [17] and $73 \%$ at 5 years [22]). A trial comparing HDR-BT given in a single 19 Gy fraction vs. two $13.5 \mathrm{~Gy}$ fractions by Morton et al. found a statistically significant difference in biochemical relapse between the two arms (7 relapses in the $19 \mathrm{~Gy}$ arm and 1 relapse in the $13.5 \mathrm{~Gy}$ $\times 2$ fractions arm at a median follow-up of 27 months) [25]. We eagerly await additional results from this trial [19]. Prada et al. subsequently increased their single fraction HDR monotherapy dose to 20.5 Gy and observed better BFFS (82\% at 6 years) [18]. We observed 3 failures in our study, all of which occurred in patients receiving 19 Gy, which may explain the suboptimal findings. After the publication by Prada et al. [17], the prescription 
Table 4. Results of other studies of single-fraction HDR-BT monotherapy and multi-fraction regimens

\begin{tabular}{|c|c|c|c|c|c|c|c|c|}
\hline Study & Regimen & $\begin{array}{l}\text { Risk } \\
\text { groups }\end{array}$ & $n$ & $\begin{array}{l}\text { Acute GU } \\
\text { toxicity }\end{array}$ & $\begin{array}{l}\text { Acute GI } \\
\text { toxicity }\end{array}$ & $\begin{array}{l}\text { Late GU } \\
\text { toxicity }\end{array}$ & $\begin{array}{l}\text { Late GI } \\
\text { toxicity }\end{array}$ & BFFS \\
\hline $\begin{array}{l}\text { Zaorsky et al. } \\
\text { (review) }\end{array}$ & LDR & NA & NA & $\begin{array}{c}>5 \% \text { grade } 3 \\
\text { or higher }\end{array}$ & $\begin{array}{c}1-5 \% \text { grade } 3 \\
\text { or higher }\end{array}$ & $\begin{array}{c}1-5 \% \text { grade } 3 \\
\text { or higher }\end{array}$ & $\begin{array}{c}<1 \% \text { grade } 3 \\
\text { or higher }\end{array}$ & $85-94 \%$ at 5 years \\
\hline Current study & $\begin{array}{c}19 \text { or } 21 \text { Gy } \\
\times 1\end{array}$ & LIR & 28 & $\begin{array}{c}14 \% \text { grade } 2 \\
\text { or higher }\end{array}$ & $\begin{array}{c}0 \% \text { grade } 2 \\
\text { or higher }\end{array}$ & $\begin{array}{c}18 \% \text { grade } 2 \\
\text { or higher }\end{array}$ & $\begin{array}{c}0 \% \text { grade } 2 \\
\text { or higher }\end{array}$ & $80.6 \%$ at 3 years \\
\hline $\begin{array}{l}\text { Prada et al. } \\
2012,2016\end{array}$ & 19 Gy × 1 & LIR & 60 & $\begin{array}{c}0 \% \text { grade } 2 \\
\text { or higher }\end{array}$ & $\begin{array}{c}0 \% \text { grade } 2 \\
\text { or higher }\end{array}$ & $\begin{array}{c}0 \% \text { grade } 2 \\
\text { or higher }\end{array}$ & $\begin{array}{c}0 \% \text { grade } 2 \\
\text { or higher }\end{array}$ & $66 \%$ at 6 years \\
\hline $\begin{array}{l}\text { Krauss et al. } \\
\text { 2017, Siddiqui } \\
\text { et al. } 2019\end{array}$ & 19 Gy × 1 & LIR & 68 & $\begin{array}{c}12 \% \text { grade } 2 \\
\text { or higher }\end{array}$ & $\begin{array}{c}0 \% \text { grade } 2 \\
\text { or higher }\end{array}$ & $\begin{array}{c}15 \% \text { grade } 2 \\
\text { or higher }\end{array}$ & $\begin{array}{l}6 \% \text { grade } 2, \\
1 \% \text { grade } 3\end{array}$ & $73 \%$ at 5 years \\
\hline \multirow[t]{2}{*}{$\begin{array}{l}\text { Morton et al. } \\
2017(a, b)\end{array}$} & $13.5 \mathrm{~Gy} \times 2$ & LIR & 83 & $\begin{array}{c}>49 \% \text { grade } 2 \\
\text { or higher }\end{array}$ & $\begin{array}{c}1 \% \text { grade } 2 \\
\text { or higher }\end{array}$ & $\begin{array}{c}27 \% \text { grade } 2 \\
\text { or higher }\end{array}$ & $\begin{array}{c}1 \% \text { grade } 2 \\
\text { or higher }\end{array}$ & $\begin{array}{l}1 \text { failure at median } \\
\text { of } 27 \text { months }\end{array}$ \\
\hline & 19 Gy × 1 & LIR & 87 & $\begin{array}{c}>49 \% \text { grade } 2, \\
1 \% \text { grade } 3\end{array}$ & $\begin{array}{c}2 \% \text { grade } 2 \\
\text { or higher }\end{array}$ & $\begin{array}{c}30 \% \text { grade } 2 \\
\text { or higher }\end{array}$ & $\begin{array}{c}3 \% \text { grade } 2 \\
\text { or higher }\end{array}$ & $\begin{array}{l}7 \text { failures at medi- } \\
\text { an of } 27 \text { months }\end{array}$ \\
\hline $\begin{array}{l}\text { Prada et al. } \\
2018\end{array}$ & 20.5 Gy × 1 & LIR & 60 & $\begin{array}{c}0 \% \text { grade } 2 \\
\text { or higher }\end{array}$ & $\begin{array}{c}0 \% \text { grade } 2 \\
\text { or higher }\end{array}$ & $\begin{array}{c}0 \% \text { grade } 2 \\
\text { or higher }\end{array}$ & $\begin{array}{c}0 \% \text { grade } 2 \\
\text { or higher }\end{array}$ & $82 \%$ at 6 years \\
\hline $\begin{array}{l}\text { Gomez-Iturria- } \\
\text { ga et al. } 2018\end{array}$ & 19 Gy × 1 & LIR & 43 & $\begin{array}{c}9 \% \text { grade } 2 \\
\text { or higher }\end{array}$ & $\begin{array}{c}0 \% \text { grade } 2 \\
\text { or higher }\end{array}$ & $\begin{array}{c}7 \% \text { grade } 2 \\
\text { or higher }\end{array}$ & $\begin{array}{c}0 \% \text { grade } 2 \\
\text { or higher }\end{array}$ & NA \\
\hline \multirow[t]{3}{*}{$\begin{array}{l}\text { Hoskin et al. } \\
2014\end{array}$} & 13 Gy $\times 2$ & $\begin{array}{c}\text { locally } \\
\text { advanced }\end{array}$ & 115 & $\begin{array}{c}13 \% \text { grade } 2, \\
6 \% \text { grade } 3\end{array}$ & $\begin{array}{c}3 \% \text { grade } 2 \\
\text { or higher }\end{array}$ & $\begin{array}{c}3 \% \text { grade } 2 \\
\text { or higher }\end{array}$ & $\begin{array}{c}1 \% \text { grade } 2 \\
\text { or higher }\end{array}$ & NA \\
\hline & 19 Gy × 1 & $\begin{array}{c}\text { locally } \\
\text { advanced }\end{array}$ & 24 & $\begin{array}{c}0 \% \text { grade } 2 \\
\text { or higher }\end{array}$ & $\begin{array}{c}0 \% \text { grade } 2 \\
\text { or higher }\end{array}$ & $\begin{array}{c}0 \% \text { grade } 2 \\
\text { or higher }\end{array}$ & $\begin{array}{c}0 \% \text { grade } 2 \\
\text { or higher }\end{array}$ & NA \\
\hline & 20 Gy $\times 1$ & $\begin{array}{c}\text { locally } \\
\text { advanced }\end{array}$ & 26 & $\begin{array}{l}0 \% \text { grade } 2, \\
9 \% \text { grade } 3\end{array}$ & $\begin{array}{c}0 \% \text { grade } 2 \\
\text { or higher }\end{array}$ & $\begin{array}{l}0 \% \text { grade } 2, \\
9 \% \text { grade } 3\end{array}$ & $\begin{array}{c}5 \% \text { grade } 2 \\
\text { or higher }\end{array}$ & NA \\
\hline \multirow[t]{3}{*}{$\begin{array}{l}\text { Hoskin et al. } \\
2017\end{array}$} & $10.5 \mathrm{~Gy} \times 3$ & $\begin{array}{c}\text { locally } \\
\text { advanced }\end{array}$ & 106 & NA & NA & $\begin{array}{l}2 \% \text { grade } 2, \\
3 \% \text { grade } 3\end{array}$ & $\begin{array}{c}2 \% \text { grade } 2 \\
\text { or higher }\end{array}$ & $90 \%$ at 4 years \\
\hline & $13 \mathrm{~Gy} \times 2$ & $\begin{array}{c}\text { locally } \\
\text { advanced }\end{array}$ & 138 & NA & NA & $\begin{array}{l}2 \% \text { grade } 2, \\
1 \% \text { grade } 3\end{array}$ & $\begin{array}{c}4 \% \text { grade } 2 \\
\text { or higher }\end{array}$ & $91 \%$ at 4 years \\
\hline & $\begin{array}{c}19 \text { or } 20 \text { Gy } \\
\times 1\end{array}$ & $\begin{array}{c}\text { locally } \\
\text { advanced }\end{array}$ & 49 & NA & NA & $\begin{array}{l}2 \% \text { grade } 2, \\
2 \% \text { grade } 3\end{array}$ & $\begin{array}{c}0 \% \text { grade } 2 \\
\text { or higher }\end{array}$ & $88 \%$ at 4 years \\
\hline
\end{tabular}

LDR - low dose rate, LIR - low to intermediate risk, GU - genitourinary, GI - gastrointestinal, BFFS - biochemical failure-free survival.

dose at our institution was increased to $21 \mathrm{~Gy}$, which was received by 2 patients reported in this series. While data from Prada et al. using 20.5 Gy [18] are encouraging, the biochemical control is still somewhat less than LDR (Table 4), and additional data and monitoring will be required. To our knowledge, the only other studies reporting single fraction HDR brachytherapy > 19 Gy are trials involving locally advanced prostate cancer patients, which compare $20 \mathrm{~Gy} \times 1$ and $19 \mathrm{~Gy} \times 1$ to multi-fraction regimens $[15,16]$. The rates of BFFS were found to be comparable to multi-fraction regimens.

The suboptimal biochemical control observed in the present study may be related to suboptimal dose delivery and/or suboptimal dose coverage. In Table 3 and Figure 4 , the details of the 3 patients who failed are provided. The coverage of the prostate base in patient 2 was suboptimal, especially on the left side, and this is where the C-11 PET showed signs of recurrence. Patient 3 had excellent coverage of the prostate base. The fact that the left posterior lateral prostate, right in the center of two catheters, had evidence of recurrence on the C11-PET suggests that the dose was suboptimal. There was also evidence of disease anterior and lateral to the proximal seminal vesicle, which is likely due to extension from the recurrence rather than suboptimal coverage in an area we would not routinely treat given that there was no seminal vesicle extension or extraprostatic spread on MRI. Patient 1 had the best implant of all the failures. Of note, prior to the implant there was a discrepancy between a CT that showed sclerotic lesions in the right ilium and ischium and a negative bone scan. While metastatic disease is a possibility, this is less likely given that the sclerotic lesions remained stable two years later with an additional negative bone scan.

Regarding the potential need for CTV expansion, 15 of the 16 patients who underwent MRI had no evidence of extraprostatic extension, and one had suspicion of extension into the preprostatic fat. While CTV expansion should perhaps be considered in such cases, the latter patient has a consistently declining PSA (from 7 to $0.25 \mathrm{ng} / \mathrm{dl})$.

The $\mathrm{EQD}_{2}$ given $\alpha / \beta=1.5$ for 19 and 21 Gy HDR-BT delivered in a single fraction is 111.3 and $135 \mathrm{~Gy}$, respectively, using the formula $\mathrm{EQD}_{2}=$ total dose * (dose per fraction $+\alpha / \beta) /(2+\alpha / \beta)$. This dose compares favorably to the 74-80 Gy delivered in the standard 2 Gy/fraction, and the dose is similar to other hypofractionated HDR-BT 

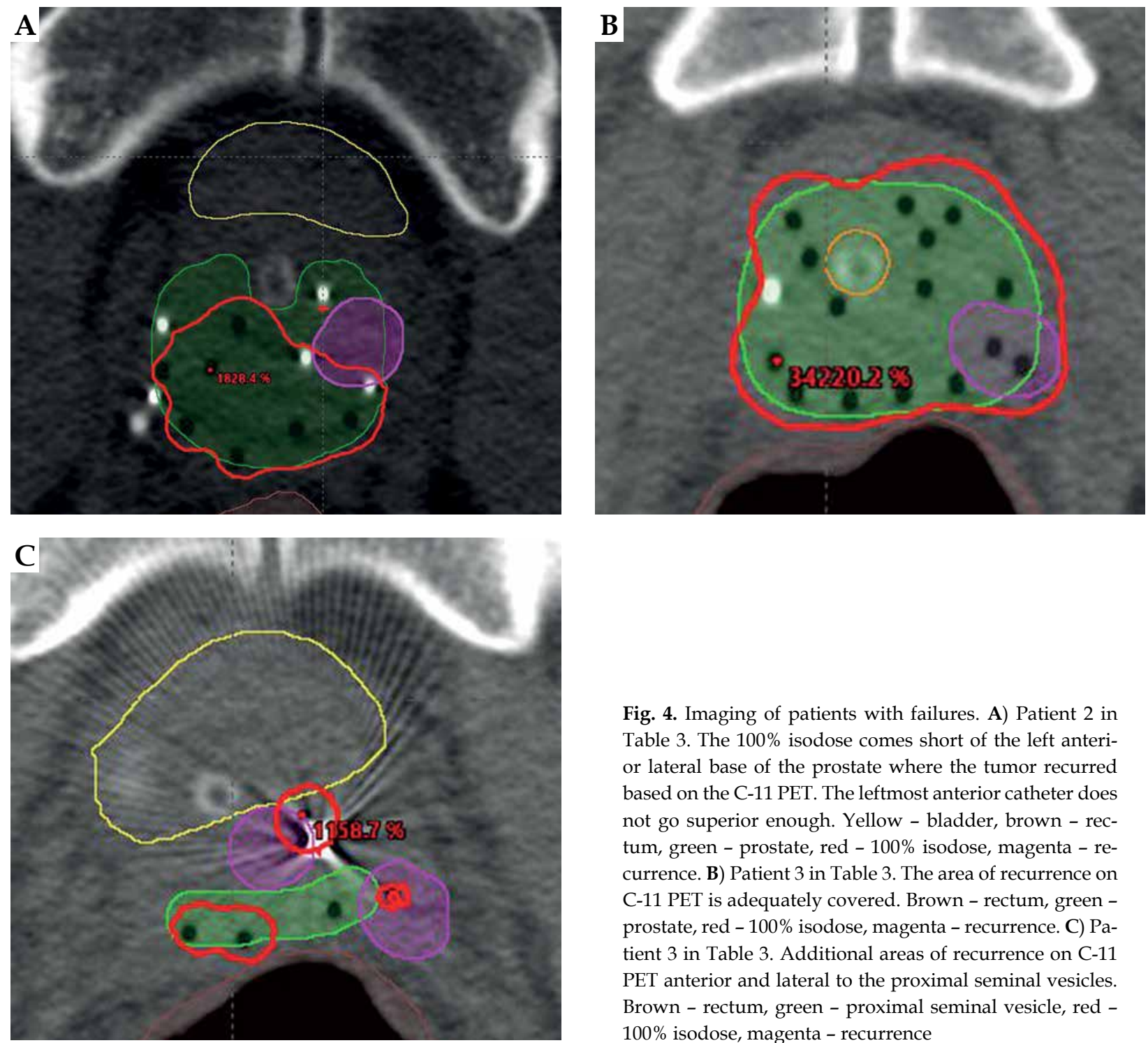

Fig. 4. Imaging of patients with failures. A) Patient 2 in Table 3. The $100 \%$ isodose comes short of the left anterior lateral base of the prostate where the tumor recurred based on the C-11 PET. The leftmost anterior catheter does not go superior enough. Yellow - bladder, brown - rectum, green - prostate, red $-100 \%$ isodose, magenta - recurrence. B) Patient 3 in Table 3. The area of recurrence on C-11 PET is adequately covered. Brown - rectum, green prostate, red $-100 \%$ isodose, magenta - recurrence. C) Patient 3 in Table 3. Additional areas of recurrence on C-11 PET anterior and lateral to the proximal seminal vesicles. Brown - rectum, green - proximal seminal vesicle, red $100 \%$ isodose, magenta - recurrence

monotherapy regimens of $38 \mathrm{~Gy}$ in 4 fractions $\left(\mathrm{EQD}_{2}=\right.$ $119.4)$, and $27 \mathrm{~Gy}$ in 2 fractions $\left(\mathrm{EQD}_{2}=115.7\right)$. Siddiqui et al. provide an excellent discussion of potential reasons for the suboptimal outcomes, including the possibility that the effective $\alpha / \beta$ is greater than 1.5 , which would result in lower-than-predicted $\mathrm{EQD}_{2}$, or that the classic linear-quadratic (LQ) equation inadequately models single-fraction regimens [22].

We found no significant change in symptom scores in the period surrounding HDR-BT and that symptom scores following treatment decreased over time. Two other studies examined trends in symptom scores in LIR-PC patients treated with single fraction HDR brachytherapy as monotherapy $[19,21]$. Morton et al. found that patients experienced worsened International Prostate Symptom Score (IPSS) for the first 12 months relative to baseline and worsened urinary and sexual quality of life EPIC scores at 6 and 12 months compared to baseline [19]. Gomez-Iturriaga et al. found no change in health-related quality of life in any domain based on EPIC scores [21].
The observed differences may be related to the nature of the questionnaires as well as the retrospective nature of the present study. A prospective study needs to be performed to better assess patient-reported outcomes. For single fraction HDR-BT for locally advanced prostate cancer, trends in moderate and severe IPSS scores were relatively flat over the first 12 weeks following therapy, though patients receiving 20 vs. 19 Gy may have had worse scores at 2 weeks [15]. The prevalence of moderate and severe IPSS scores declined from approximately $30 \%$ to $20 \%$ and from $10 \%$ to $5 \%$ at 24 and 48 months, respectively [16], which is similar to the prevalence of moderate and severe AUA scores at 24 months $(36 \%$ and $14 \%$, respectively) in our study.

Some have questioned the future of prostate HDR-BT as monotherapy given the results of $19 \mathrm{~Gy} \times 1$. However, results from Prada et al. with 19 Gy and 20.5 Gy suggest a dose response, as a $1.5 \mathrm{~Gy}$ increase in dose improved the actuarial biochemical control by $16 \%[17,18]$. Assuming a linear relationship, another increase in 1.5 Gy may result 
in over $95 \%$ biochemical control at 6 years. Our institution has a prospective trial of 21 Gy HDR-BT monotherapy, but based on the available evidence we are increasing the dose to $23 \mathrm{~Gy}$. While the LQ model is reasonable for initially selecting doses for clinical trials, it is through clinical trials that clinically effective doses are determined.

Limitations of the present study include the relatively small number of patients, the lack of extended follow-up needed to more accurately understand clinical outcomes, and the retrospective nature of the study design. Furthermore, the 19 Gy dose may be insufficient for proper disease control. To address these issues, our institution has started a prospective phase II trial, NCT03424850, to evaluate toxicities and outcomes of 21 Gy HDR brachytherapy delivered in a single fraction for LIR-PC.

\section{Conclusions}

In conclusion, our early data suggest that single fraction HDR-BT monotherapy for patients with LIR-PC has limited rates of radiation-related toxicities. However, it has suboptimal clinical outcomes with lower than expected 3-year BFFS. While we report similar toxicities and biochemical control to other studies, the prospective trial currently underway with $21 \mathrm{~Gy}$ in a single fraction hopefully will result in improved outcomes with acceptable toxicity. However, given the suboptimal biochemical control observed in this and other studies, single fraction HDR-BT should not be considered a standard treatment option for LIR-PC and should be performed only in the context of a prospective trial.

Research was conducted at the Department of Radiation Oncology at Washington University.

\section{Disclosure}

The authors report no conflict of interest.

\section{References}

1. Michalski JM, Moughan J, Purdy J et al. Effect of standard vs dose-escalated radiation therapy for patients with intermediate-risk prostate cancer. JAMA Oncol 2018; 4: e180039.

2. Heemsbergen WD, Al-Mamgani A, Slot A et al. Phase III randomised trial Long-term results of the Dutch randomized prostate cancer trial: Impact of dose-escalation on local, biochemical, clinical failure, and survival. Radiother Oncol 2014; 110: 104-109.

3. Zietman AL, Bae K, Slater JD et al. Randomized trial comparing conventional-dose with high-dose conformal radiation therapy in early-stage adenocarcinoma of the prostate: long-term results from proton radiation oncology group/ American college of radiology 95-09. J Clin Oncol 2010; 28: 1106-1111.

4. Kuban DA, Tucker SL, Dong L et al. Long-term results of the M. D. Anderson randomized dose-escalation trial for prostate cancer. Int J Radiat Oncol 2008; 70: 67-74.

5. Dearnaley DP, Jovic G, Syndikus I et al. Escalated-dose versus control-dose conformal radiotherapy for prostate cancer: long-term results from the MRC RT01 randomised controlled trial. Lancet Oncol 2014; 15: 464-473.

6. Demanes DJ, Ghilezan MI. High-dose-rate brachytherapy as monotherapy for prostate cancer. Brachytherapy 2014; 13 529-541.
7. Zaorsky NG, Davis BJ, Nguyen PL et al. The evolution of brachytherapy for prostate cancer. Nat Rev Urol 2017; 14: 415-439.

8. Hoskin P. High dose rate brachytherapy for prostate cancer. Cancer Radiother 2008; 12: 512-514.

9. Thompson I, Thrasher JB, Aus G et al. Guideline for the management of clinically localized prostate cancer: 2007 update. J Urol 2007; 177: 2106-2131.

10. Galalae RM, Kovács G, Schultze J et al. Long-term outcome after elective irradiation of the pelvic lymphatics and local dose escalation using high-dose-rate brachytherapy for locally advanced prostate cancer. Int J Radiat Oncol Biol Phys 2002; 52: 81-90.

11. Galalae RM, Martinez A, Mate T et al. Long-term outcome by risk factors using conformal high-dose-rate brachytherapy (HDR-BT) boost with or without neoadjuvant androgen suppression for localized prostate cancer. Int J Radiat Oncol 2004; 58: 1048-1055.

12. Galalae RM, Zakikhany NH, Geiger F et al. The 15-year outcomes of high-dose-rate brachytherapy for radical dose escalation in patients with prostate cancer - a benchmark for high-tech external beam radiotherapy alone? Brachytherapy 2014; 13: 117-122.

13. Yoshioka $\mathrm{Y}$, Nose $\mathrm{T}$, Yoshida $\mathrm{K}$ et al. High-dose-rate interstitial brachytherapy as a monotherapy for localized prostate cancer: treatment description and preliminary results of a phase I/II clinical trial. Int J Radiat Oncol Biol Phys 2000; 48: 675-681.

14. Prada PJ, Jimenez I, González-Suárez H et al. High-dose-rate interstitial brachytherapy as monotherapy in one fraction and transperineal hyaluronic acid injection into the perirectal fat for the treatment of favorable stage prostate cancer: treatment description and preliminary results. Brachytherapy 2012; 11: 105-110.

15. Hoskin P, Rojas A, Ostler P et al. High-dose-rate brachytherapy alone given as two or one fraction to patients for locally advanced prostate cancer: Acute toxicity. Radiother Oncol 2014; 110: 268-271.

16. Hoskin P, Rojas A, Ostler P et al. Prostate brachytherapy single-dose high-dose-rate brachytherapy compared to two and three fractions for locally advanced prostate cancer. Radiother Oncol 2017; 124: 56-60.

17. Prada PJ, Cardenal J, Blanco AG et al. High-dose-rate interstitial brachytherapy as monotherapy in one fraction for the treatment of favorable stage prostate cancer: Toxicity and long-term biochemical results. Radiother Oncol 2016; 119: 411416.

18. Prada PJ, Ferri M, Cardenal J et al. High-dose-rate interstitial brachytherapy as monotherapy in one fraction of $20.5 \mathrm{~Gy}$ for the treatment of localized prostate cancer: Toxicity and 6-year biochemical results. Brachytherapy 2018; 17: 845-851.

19. Morton G, Chung HT, Mcguffin M et al. Phase II randomized trial prostate high dose-rate brachytherapy as monotherapy for low and intermediate risk prostate cancer: Early toxicity and quality-of life results from a randomized phase II clinical trial of one fraction of $19 \mathrm{~Gy}$ or two fractions of $13.5 \mathrm{~Gy}$. Radiother Oncol 2017a; 122: 87-92.

20. Krauss DJ, Ye H, Martinez AA et al. Favorable preliminary outcomes for men with low- and intermediate-risk prostate cancer treated with 19-gy single-fraction high-dose-rate brachytherapy. Int J Radiat Oncol 2017; 97: 98-106.

21. Gomez-Iturriaga A, Casquero F, Pijoan JI et al. Health-related-quality-of-life and toxicity after single fraction 19 Gy high-dose-rate prostate brachytherapy: Phase II trial. Radiother Oncol 2018; 126: 278-282.

22. Siddiqui ZA, Gustafson GS, Ye H et al. 5-year outcomes of a single institution prospective trial of 19 gy single-fraction 
HDR brachytherapy for low- and intermediate-risk prostate cancer. Int J Radiat Oncol 2019; 104: 1038-1044.

23. Schroeder C, Geiger F, Siebert FA et al. Radical dose escalation by high-dose-rate brachytherapy for localized prostate cancer - significance of prostate-specific antigen nadir level within 18 months as correlation for long-term biochemical control. Brachytherapy 2019; 18: 8-12.

24. Zaorsky NG, Shaikh T, Murphy CT et al. Comparison of outcomes and toxicities among radiation therapy treatment options for prostate cancer. Cancer Treat Rev 2016; 48: 50-60.

25. Morton G, Chung H, McGuffin M et al. Prostate HDR monotherapy: initial efficacy results from a randomized trial of one versus two fractions. Brachytherapy 2017b; 16: S19-S20. 